\title{
Expression of methylation-related genes is associated with overall survival in patients with non-small cell lung cancer
}

\author{
J Xing', DJ Stewart', J Gu', C Lu², MR Spitz' and X Wü,I \\ 'Department of Epidemiology, Unit 1340, The University of Texas MD Anderson Cancer Center, I I 55 Pressler Boulevard, Houston, TX 77030, USA; \\ ${ }^{2}$ Department of Thoracic/Head \& Neck Medical Oncology, The University of Texas MD Anderson Cancer Center, Houston, TX 77030, USA
}

The abnormality of DNA methylation is involved in tumour progression, and thus has a modulating effect on clinical outcome of cancer patients. In this study, we measured the mRNA expression levels of three methylation-regulating genes (DNMTI, DNMT3b, and MBD2) in 148 tumour samples from patients with non-small cell lung cancer (NSCLC) using quantitative real-time polymerase chain reaction and then determined their prognostic values. Our data showed that the high level of DNMTI expression was significantly associated with an increased risk of death in all NSCLC patients (hazard ratio (HR), I.74; 95\% confidence interval (95\% Cl), 1.04-2.90). However, the high level of DNMT3b expression was significantly associated with poor prognosis only in young patients (<65 years). The high level of MBD2 expression had a significantly reduced risk for death only in male patients and in squamous cell lung carcinoma (SQLC) patients. All three combination groups with DNMTI and DNMT3b, DNMTI and MBD2 or DNMT3b and MBD2 revealed significant combined effects in male patients and SQLC patients. Our results suggest that DNMTI, DNMT3b, and MBD2 may play important roles in modulating NSCLC patient survival and thus be useful for identifying NSCLC patients who would benefit most from aggressive therapy.

British Journal of Cancer (2008) 98, 1716-1722. doi:10.1038/sj.bjc.6604343 www.bjcancer.com

Published online 15 April 2008

(C) 2008 Cancer Research UK

Keywords: DNA methyltransferase; hypermethylation; non-small cell lung cancer, prognosis

The malignant transformation of human cells is usually driven by the activation of oncogenes or the inactivation of tumour suppressor genes, which can be caused by epigenetic events (Barrett et al, 1986; Simons, 1995; Jones and Laird, 1999). DNA methylation has been well documented as the major form of epigenetic gene regulation (Shames et al, 2007). Furthermore, an altered DNA methylation pattern is one of the most consistent epigenetic hallmarks of human cancer (Baylin, 2005; Esteller, 2005; Luczak and Jagodzinski, 2006). Usually, neoplastic cells simultaneously exhibit global genomic hypomethylation and regionspecific hypermethylation. Most hypomethylation events in cancer cells appear to occur in repetitive and parasitic elements, which are heavily methylated in normal cells (Hoffmann and Schulz, 2005). These events may result in increased genomic instability. The hypermethylation of $\mathrm{CpG}$ islands in the promoter region of genes has been reported to cause the inactivation of tumour suppressor genes, which is critical to the process of tumorigenesis (NewellPrice et al, 2000; Teodoridis et al, 2004; McCabe and Caudill, 2005).

The exact nature of the defect in methylation machinery of tumour cells remain unclear; however, it may be related to the expression of DNA methyltransferases (DNMTs), primarily DNMT1, DNMT3a, and DNMT3b (Leonhardt and Bestor, 1993). DNMTs usually catalyse the transfer of methyl groups to CpG dinucleotides to produce either hemimethylated or fully

*Correspondence: Dr X Wu; E-mail: xwu@mdanderson.org

Received 24 September 2007; revised 18 January 2008; accepted 10 March 2008; published online 15 April 2008 methylated DNA strands. DNMT1 is best known as the maintenance methyltransferase that copies methylation patterns after DNA replication; DNMT3a and DNMT3b are primarily de novo methylators of CpG sites. Numerous studies (Issa et al, 1993; Robertson et al, 1999) have evaluated the expression of DNMTs in tumour tissue, and most have reported variable levels of overexpression, particularly of DNMT1 and DNMT3b. Rhee et al (2002) demonstrated that DNMT1 and DNMT3b cooperatively maintain DNA methylation and gene silencing in human cancer cells. In contrast, DNA demethylase-methyl-CpG-binding domain 2 (MBD2) performs the reverse reaction to DNMTs (Bhattacharya et al, 1999). Compared to the adjacent normal tissue, a significant decrease in $M B D 2$ mRNA expression has been observed in various tumour tissue types (Kanai et al, 1999; Patra et al, 2002). These findings suggest that DNA methyltransferases and DNA demethylases play pivotal roles in the initiation and progression of tumours and thus may be useful in the clinical diagnosis and prognostic assessment of cancer.

Lung cancer is the leading cause of cancer-related death in the United States, with an estimated 160390 deaths in 2007 (Jemal et al, 2007). The most common histological type is non-small cell lung cancer. The current 5-year survival rate of non-small cell lung cancer (NSCLC) is only $2-47 \%$ for different stages, and this has improved little over the past two decades. Thus, new prognostic markers are needed to help identify patients with poor prognoses, who may benefit from more aggressive treatment approaches. Previous studies have consistently reported that DNMT isoforms are significantly upregulated in human lung cancer cell lines and NSCLC tissue specimens (Sato et al, 2002; Vallbohmer et al, 2006). 
However, studies of the association between DNMT expression and clinical outcome in NSCLC patients have produced inconsistent results (Kim et al, 2006; Vallbohmer et al, 2006; Lin et al, 2007), and few have evaluated the prognostic value of $M B D 2$. Therefore, we determined the expression of DNMT1, DNMT3b, and MBD2 by real-time quantitative PCR in 148 tumour samples from NSCLC patients and analysed their prognostic value, both separately and jointly.

\section{MATERIALS AND METHODS}

\section{Patients and tissue specimens}

One hundred forty-eight patients with histologically confirmed NSCLC were included in this study, all of whom were recruited from 1993 to 1997 and had undergone curative surgical resections at The University of Texas MD Anderson Cancer Center. There were no age, sex, ethnicity, or tumour stage restrictions on patient enrolment. Fresh tumour tissues were frozen immediately after excision and stored in liquid nitrogen until they were used for RNA extraction. Demographic and clinical data were collected from the patient history database and chart review at MD Anderson Cancer Center. The survival duration was evaluated as of June 2005. Study approval was obtained from the MD Anderson institutional review board.

\section{RNA extraction and cDNA synthesis}

Total RNA extraction and cDNA synthesis were performed as previously described (Lin et al, 2006). In brief, frozen tissues were homogenised, and total RNA was isolated with the EZNA total RNA kit (Omega Bio-tek, Doraville, GA, USA) according to the manufacturer's instructions. RNA was eluted in RNase-free water, and the concentration was determined by spectrophotometer (Beckman Coulter, Fullerton, CA, USA). The quality of RNA samples was determined by $1 \%$ agarose gel electrophoresis and ethidium bromide-staining. The cDNA synthesis reaction was then carried out using the Taqman reverse transcription reagents kit (Applied Biosystems, Branchburg, NJ, USA) in a final volume of $20 \mu$ containing $1 \times \mathrm{RT}$ buffer, $5 \mathrm{mmoll}^{-1} \mathrm{MgCl}_{2}, 250 \mu \mathrm{moll}^{-1}$ each dNTP, 20 units of RNase inhibitor, 50 units of multiscribe reverse transcriptase, $2.5 \mu \mathrm{moll}^{-1}$ random hexamers, and $0.5 \mu \mathrm{g}$ of total RNA. The reaction mixtures were incubated at ambient temperature for $10 \mathrm{~min}$ and then at $42^{\circ} \mathrm{C}$ for $30 \mathrm{~min}$. Reverse transcriptase was inactivated by heating at $99^{\circ} \mathrm{C}$ for $5 \mathrm{~min}$. All cDNA products were stored at $-30^{\circ} \mathrm{C}$ until they were used for the real-time PCR.

\section{Real-time PCR}

The primers and probes for real-time PCR were designed using Primer Express software (version 2.0, Applied Biosystems). We confirmed the specificity of primers and probes and the absence of single nucleotide polymorphisms by searching the Genebank database. To avoid amplifying residual genomic DNA, one of the two primers or the probe was designed across the junction region between two exons. The sequences of the primers and probes were as follows: DNMT1, forward primer (FP): 5-AGAAGAGACGTAGA GTTACATCCAGAGA-3, reverse primer (RP): 5-GCGTTCCTGATT TTGCTCTTTC-3, probe: 5-FAM- CGAGTTGCTAGACCGCTTCCT GC AGA-TAMRA-3; DNMT3b, FP: 5-CGCACCCCGGAGATCA-3, RP: 5-ACTGGACACCTCCC TCTTGGA-3, probe: 5-FAM-AGGCCG AAGATCAAGCTCGCGACT-TAMRA-3; MBD2, FP: 5-AGTGAAAT CAGACCCACAACGA-3, RP: 5-GCACTAAGTCCT TGTAGCCTCTT CTC-3, probe: 5-FAM-TGAATGAACAGCCACGTCAGCTTTTCTA TAMRA-3; and GAPDH, FP: 5-AAGGCTGAGAACGGGAAGC-3, RP: 5-GAGGGATCTCGC TCCTGGA-3, probe: 5-FAM-TGTCATCA ATGGAAATCCCATCACCATC-TAMRA-3.
PCR amplification and real-time detection of product were performed using the ABI Prism 7900 sequence detection system (Applied Biosystems) in a $10-\mu \mathrm{l}$ reaction mixture consisting of $1 \times$ Taqman buffer A, $3.4 \mathrm{mM} \mathrm{MgCl}_{2}, 100 \mu \mathrm{M}$ each dNTP, $0.2 \mu \mathrm{M}$ each primer, $0.1 \mu \mathrm{m}$ probe, $0.02 \mathrm{U}$ of AmpliTaq Gold DNA polymerase, and $1 \mu \mathrm{l}$ of each synthesized cDNA template. The thermal cycling conditions comprised one cycle at $95^{\circ} \mathrm{C}$ for $10 \mathrm{~min}$ and 40 cycles at $95^{\circ} \mathrm{C}$ for $15 \mathrm{~s}, 60^{\circ} \mathrm{C}$ for $1 \mathrm{~min}$. A relative quantification method using standard curve was used to measure the relative expression levels of each gene in all samples. In brief, the cDNA product of commercial human total RNA (Stratagene, La Jolla, CA, USA) was serially diluted by fivefold per dilution to produce a 6-point standard curve for each tested gene. The quantity of tested gene in each sample was arbitrarily measured as the dilution level of standard sample. The human GAPDH gene was used as an internal control to normalise the RNA input amount, reverse transcription efficiency, and RNA quality. The relative expression levels of each sample were expressed as $\mathrm{N}$-fold expression differences in the target gene relative to the GAPDH genes. The PCR reaction for each sample was duplicated, and the mean value was used in the statistical analysis.

\section{Statistical analysis}

We used STATA statistical software, version 8.0 (Stata Corp., College Station, TX, USA), for all statistical analyses. Smoking status and pack-years were categorised as previously described (Wu et al, 2003). Overall survival duration was defined as the time from lung cancer diagnosis to the date of patient death or last follow-up. Survival status was evaluated using Pearson's $\chi^{2}$ test or Fisher's exact test for categorical variables and Student's $t$-test for continuous variables. The expression levels of all three genes were dichotomised as high or low, with the median values of normalised mRNA expression used as the cutoff points. The hazard ratios and $95 \%$ confidence intervals were calculated using Cox proportional hazards regression analysis to determine the effects of gene expression level on overall survival. Multivariate analysis was used to control potential confounding factors (age, sex, ethnicity, smoking status, tumour grade, and clinical disease stage). KaplanMeier plots and the log-rank test were used to evaluate the association between survival duration and the expression of the three genes. Because only three genes were involved in our study, multiple testing issues have not been considered for our analysis. All reported $P$-values were based on two-sided tests, and the level of significance was set at $P<0.05$.

\section{RESULTS}

\section{Distribution of demographic and clinical variables}

Patients' demographic and clinical characteristics are summarised in Table 1. One hundred forty-eight NSCLC patients were included in this study, with a mean age of 65.0 years. Ninety-four percent of patients were white, and $92 \%$ were ever smokers. The most common histologic tumour types were lung adenocarcinoma (47\%) and SQLC (36\%). Forty-eight percent of patients were at stage I, $15 \%$ at stage II, $23 \%$ at stage III and $14 \%$ unknown. However, tumours were mostly at intermediate (grade $2(38.5 \%)$ ) or low (grade $3(39 \%)$ ) differentiations. The median survival duration was 45.9 months, and the 5 -year overall survival rate was $42 \%$. Among 136 patients with complete treatment information, 77 (57\%) patients received surgery alone and 59 (43\%) patients received surgery with adjuvant chemo or radiotherapy. As shown in Table 1, there were no significant differences in the age $(P=0.16), \quad$ sex $\quad(P=0.22), \quad$ ethnicity $\quad(P=0.54), \quad$ smoking status $(P=0.42)$, pack-years $(P=0.66)$, or histologic tumour subtype $(P=0.79)$ between patients who were alive and dead at 
Table I Distribution of demographic and clinical variables by survival status

\begin{tabular}{|c|c|c|c|c|c|}
\hline \multirow[b]{2}{*}{ Variable } & \multicolumn{2}{|c|}{$\begin{array}{c}\text { Alive } \\
(n=49)\end{array}$} & \multicolumn{2}{|c|}{$\begin{array}{c}\text { Death } \\
(n=99)\end{array}$} & \multirow[b]{2}{*}{$P$-value } \\
\hline & Number & (\%) & Number & (\%) & \\
\hline \multicolumn{6}{|l|}{ Gender } \\
\hline Male & 24 & (49) & 59 & (60) & \\
\hline Female & 25 & $(51)$ & 40 & $(40)$ & 0.22 \\
\hline \multicolumn{6}{|l|}{ Ethnicity } \\
\hline Caucasian & 45 & $(92)$ & 94 & (95) & \\
\hline Mexican American & I & $(2)$ & 2 & $(2)$ & \\
\hline African American & 2 & (4) & 3 & (3) & \\
\hline Others & 1 & $(2)$ & 0 & $(0)$ & 0.54 \\
\hline \multicolumn{6}{|l|}{ Smoking status ${ }^{a}$} \\
\hline Never smoker & 6 & $(13)$ & 6 & $(6)$ & \\
\hline Former smoker & 17 & (38) & 36 & (40) & \\
\hline Current smoker & 22 & (49) & 49 & (54) & 0.42 \\
\hline \multicolumn{6}{|l|}{ Histology } \\
\hline Adenocarcinoma & 23 & $(47)$ & 46 & (46) & \\
\hline SQLC & 19 & (39) & 34 & (34) & \\
\hline Other & 7 & $(14)$ & 19 & (20) & 0.79 \\
\hline \multicolumn{6}{|l|}{ Tumour stage $\mathrm{e}^{\mathrm{a}}$} \\
\hline 1 & 33 & (79) & 38 & (45) & \\
\hline$\|$ & 5 & $(12)$ & 17 & (20) & \\
\hline III & 4 & $(10)$ & 30 & (35) & $<0.001$ \\
\hline \multicolumn{6}{|l|}{ Grade $^{a}$} \\
\hline 1 & 7 & $(18)$ & 5 & $(6)$ & \\
\hline$\|$ & 16 & $(4 I)$ & 41 & (46) & \\
\hline III & 16 & $(41)$ & 42 & $(48)$ & 0.09 \\
\hline \multicolumn{6}{|l|}{ Treatment } \\
\hline Surgery alone & 35 & $(45)$ & 42 & (55) & \\
\hline Surgery with adjuvant therapy & 9 & $(15)$ & 50 & (85) & $<0.001$ \\
\hline Age in years (mean \pm s.d.) & \multicolumn{2}{|c|}{$63.3 \pm 10.5$} & \multicolumn{2}{|c|}{$65.9 \pm 10.8$} & 0.16 \\
\hline Pack-years ${ }^{\text {b }}$ (mean \pm s.d.) & \multicolumn{2}{|c|}{$50.1 \pm 33.0$} & \multicolumn{2}{|c|}{$52.6 \pm 24.7$} & 0.66 \\
\hline
\end{tabular}

${ }^{a}$ Few patients did not have documented smoking status, stage or grade information at the time of this investigation. ${ }^{b}$ For ever smokers only

last follow-up. Nevertheless, the high stage at diagnosis was a significant risk predictor for death $(P<0.001)$ in all patients, followed by high grade $(P=0.09)$. In addition, when we compare patients with surgery alone, patients with adjuvant therapy had a significantly higher death rate $(P<0.001)$, mainly because adjuvant therapy, as a secondary treatment, was mostly applied to patients of high stages and at high risks of recurrence and metastasis.

\section{Association between DNMT1, DNMT3b, and MBD2 expression and overall survival}

The median expression values for DNMT1, DNMT3b, and MBD2 genes are $1.363,39.425$ and 2.978 , respectively. No significant correlation was observed between the mRNA expression of DNMT1, DNMT3b, and MBD2 and the tumour grade, stage, and histological type (data not shown). We dichotomised the expression level of these three genes into high and low groups by using the median value as the cutoff. According to the results of the multivariate Cox proportional hazards model, DNMT1 expression was significantly associated with patient survival rate of NSCLS patients. After adjusting for age, sex, ethnicity, smoking status, tumour grade, and clinical disease stage, we found that the high level of DNMT1 expression was associated with a $74 \%$ increased risk of death (HR, 1.74; 95\% CI, 1.04-2.90). However, no statistically significant association was found for DNMT3b (HR, 1.27; 95\% CI, $0.78-2.06$ ) and MBD2 (HR, 0.80; 95\% CI, $0.48-1.35$ ) (Table 2). The Kaplan-Meier analysis revealed that patients with the high level of DNMT1 expression had notably shorter median survival duration (36.8 months) than did those with the low level of expression (60.9 months) although it was not statistically significant ( $P=0.142$, log-rank test) (Figure 1A). Moreover, we found no significant difference between the median survival durations of NSCLC patients with low and high levels of DNMT3b or MBD2 expression (data not shown).

In stratified analyses (Table 2), our data showed that the high level of DNMT1 expression was associated with a high risk of death in male (HR, 2.17; 95\% CI, 1.11-4.28) but not in females (HR, 1.63; 95\% CI, 0.59-4.49), in heavy smokers (HR, 5.67; 95\% $\mathrm{CI}, 1.64-19.60)$ but not in light smokers (HR, 1.08; 95\% CI, $0.54-2.19$ ), and in SQLC patients (HR, 3.26; 95\% CI, $1.22-8.70$ ) but not in adenocarcinoma patients (HR, 1.47; 95\% CI, 0.64-3.37). The Kaplan-Meier analyses (Figure 1B-D) also showed that, compared with the low level of DNMT1 expression, the high level of expression was only notably associated with short median survival duration in males (35.7 vs 60.9 months, $P=0.102$ ), in heavy smokers (31.1 vs 61.3 months, $P=0.022)$, and in SQLC patients (31.1 vs 69.0 months, $P=0.004)$. Furthermore, a high level of DNMT3b expression was significantly associated with poor prognosis in patients aged $<65$ years (HR, 2.76; 95\% CI, 1.12-6.76). In comparison, a high level of $M B D 2$ expression provided significant protective effect in male patients ( $\mathrm{HR}, 0.40 ; 95 \% \mathrm{CI}$, $0.17-0.94$ ) and SQLC patients (HR, 0.35; 95\% CI, 0.12-1.03). The Kaplan-Meier analyses for DNMT3b and MBD2 expressions indicated the similar results (data not shown). In addition, we performed stratified analysis of the three genes by adjuvant therapy (Table 2). We didn't find any notable effects of adjuvant therapy on the prognostic values of three genes expression.

\section{Combined analysis on the prognostic value of DNMT1, DNMT3b, and MBD2 in different subgroups}

On the basis of our initial findings, we defined high expression of $D N M T 1$ and DNMT3b and low expression of MBD2 as three unfavourable factors that were associated with poor survival. We determined the combined effect of either two genes on overall survival in different subgroups of patients stratified by age, sex and histological type (Table 3). All three combination groups with $D N M T 1$ and DNMT3b, DNMT1 and MBD2 or DNMT $3 b$ and MBD2 revealed significant combined effects in male patients ( $P$ for trend $=0.023,<0.001$ and $=0.010$, respectively) and SQLC patients ( $P$ for trend $=0.022,0.003$ and 0.019 , respectively), but not in female patients ( $P$ for trend $=0.357,0.243$ and 0.341 , respectively) and adenocarcinoma patients ( $P$ for trend $=0.245$, 0.487 and 0.431 , respectively). Findings from Kaplan-Meier analyses for all three combination groups also indicated that the median overall survival duration was substantially declined as the number of unfavourable factors increased in male patients and SQLC patients but not in female patients and adenocarcinoma patients (data not shown). In comparison, we found that age exerted different effects on the prognostic value of three combination groups.

\section{DISCUSSION}

In this study, we measured the mRNA expression level of three methylation-related genes (DNMT1, DNMT3b and MBD2) in NSCLC tumour tissues using quantitative real-time PCR and evaluated their prognostic values. Our data showed that these three 
Table 2 Association of DNMTI, DNMT3b and MBD2 mRNA expression in tumour tissues with NSCLC patient survival

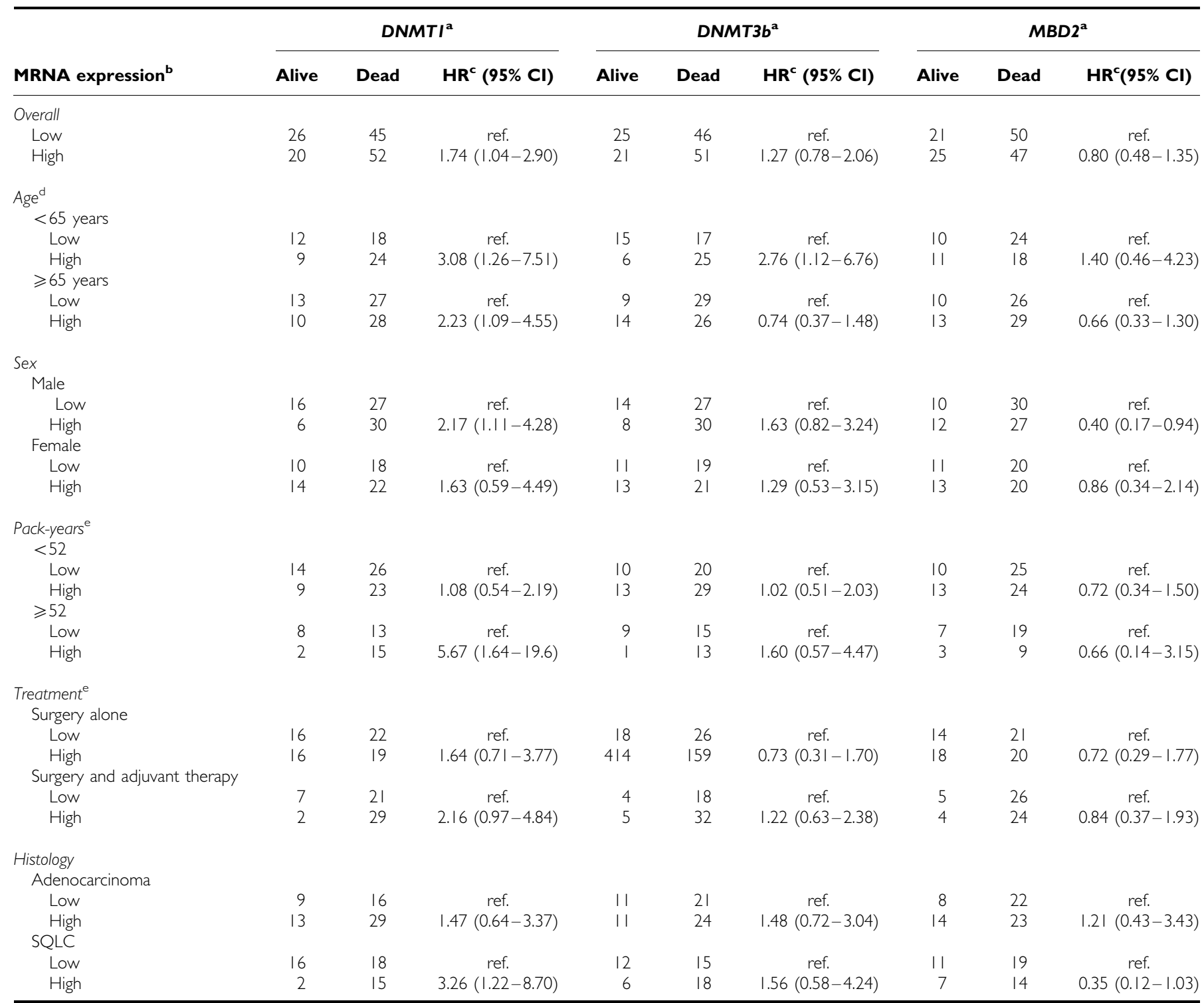

${ }^{a}$ Number of patients may not add up to the total number due to assay failure. ${ }^{b}$ Dichotomised using the median value for mRNA expression in tumour tissues. The cutoff points for DNMTI, DNMT3b, and MBD2 were 1.362, 39.425 and 2.978, respectively. ${ }^{c}$ Adjusted for age, sex, ethnicity, smoking status, tumour grade and clinical disease stage when

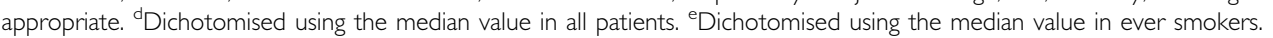

genes had modulating effects on clinical outcome of NSCLC patients.

We found that the high level of DNMT1 expression was significantly associated with poor overall survival in NSCLC patients, independent of tumour stage and grade. Kim et al (2006) reported similar results in a study of 102 NSCLC patients (HR 3.51; 95\% CI, 1.18 -12.76). Lin et al (2007) further verified the association between the high level of DNMT1 expression and poor prognosis of lung cancer at protein level. In a mouse model of prostate cancer, McCabe et al (2006) also found an association between DNMT1 and survival and demonstrated that treatment with 5-aza, a DNA methyltransferase inhibitor, prevented the development of lymph node metastases and dramatically improved survival. However, in a study of 91 NSCLC patients, no association was found between DNMT1 mRNA expression and clinical outcome (Vallbohmer et al, 2006). This discrepancy may be a result of different patient populations and different methods for normalisation of mRNA expression.
The molecular mechanism of DNMT1 survival modulation remains to be elucidated. Several previous studies (Paz et al, 2003; Robert et al, 2003) have suggested that DNMT1, as a major methylation-inducing factor, is needed to maintain CpG methylation and aberrant gene silencing in human cancer cells. Kim et al (2006) found that elevated mRNA levels of DNMT1 were significantly associated with promoter hypermethylation of tumour suppressor gene p16 in NSCLC patients. Lin et al (2007) demonstrated that DNMT1 overexpression was associated with the hypermethylation of FHIT, $p 16^{i n k 4 a}$, and $R A R \beta$ and that the promoters of methylated FHIT, $p 16^{i n k 4 a}$, and $R A R \beta$ were bound by DNMT1 protein. These findings suggest that DNMT1 hypermethylates survival-associated tumour suppressor genes, leading to their functional inactivation. However, a recent report (Sato et al, 2002) found no significant association between DNMT1 expression and DNA methylation of some tumour-associated genes, suggesting that DNMT1 also contributes to cancer development and progression through alternative pathways. For example, 

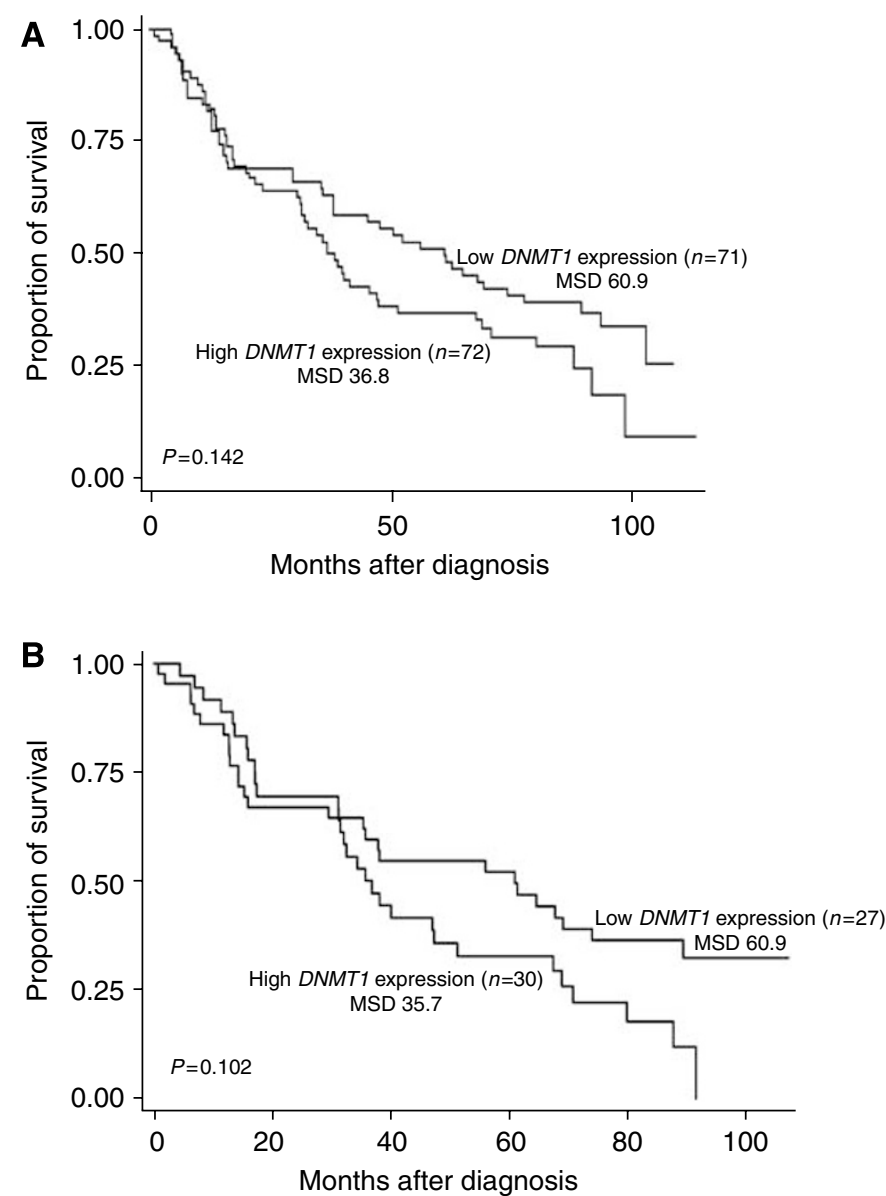
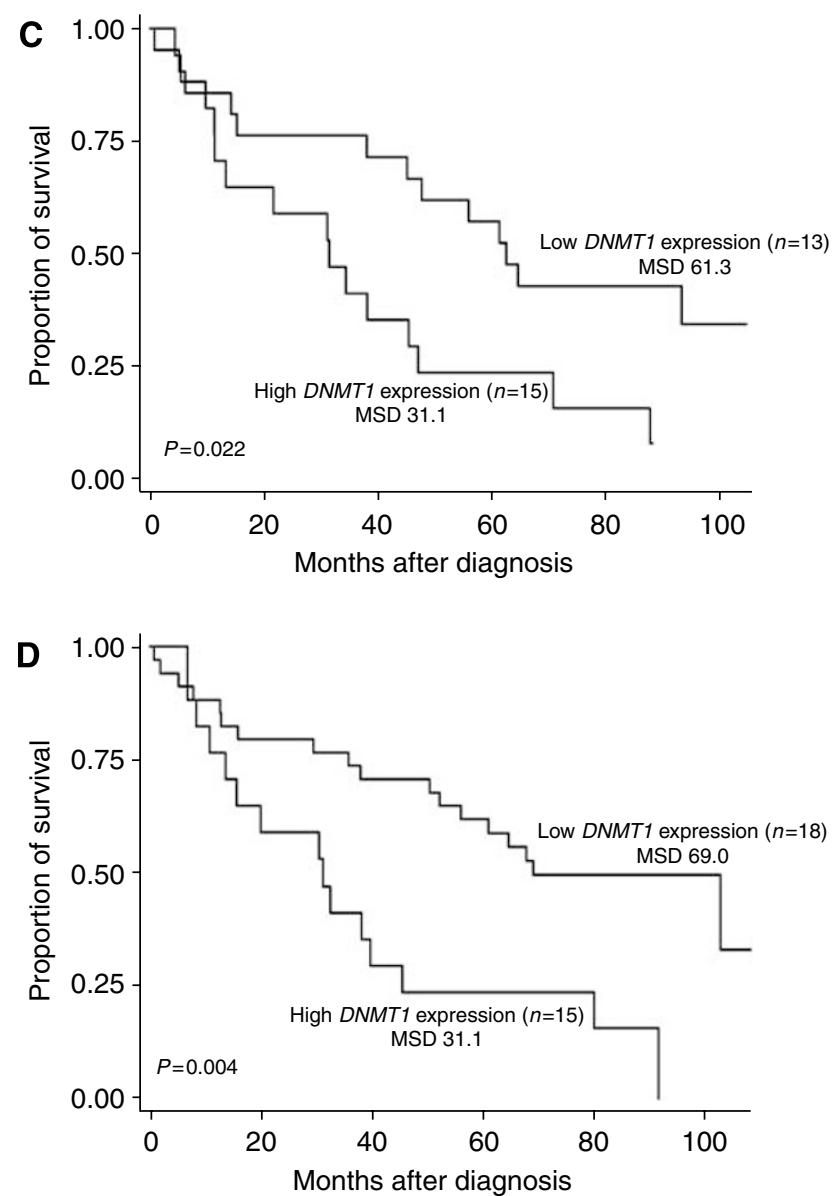

Figure I Kaplan-Meier survival estimates for patients with NSCLC. Survival duration by DNMTI expression $(\mathbf{A})$ in all patients, $(\mathbf{B})$ in male patients, $(\mathbf{C})$ in patients who were heavy smokers and (D) in SQLC patients; MSD, Median survival duration.

Chuang et al (1997) reported that DNMT1 is in a complex with proliferating cell nuclear antigen, a factor that assists in DNA replication, suggesting that DNMT1 may play a vital role in replication except for regulating gene expression. Egger et al (2006) also demonstrated that DNMT1 is essential to the proliferation and survival of cancer cells. In addition, Biniszkiewicz et al (2002) reported that the overexpression of DNMT1 resulted in the activation of silent alleles in Igf2 gene by de novo methylation, thus leading to increased cell proliferation and overgrowth.

We found no significant association between DNMT3b expression and survival in all NSCLC patients. This result is consistent with those of Kim et al (2006) and Vallbohmer et al (2006). However, possibly because of a limited number of samples, neither of these studies included an analysis stratified by patient characteristics. Our stratified data analysis revealed a significant association between the high level of $D N M T 3 b$ expression and increased risk of death in patients aged $<65$ years, suggesting that $D N M T 3 b$ expression may be an age-related prognostic predictor. Similarly, Girault et al (2003) found that DNMT3b overexpression was associated with a short relapse-free survival duration in a subgroup of breast cancer patients, and Wang et al (2004) demonstrated that increased DNMT3b promoter activity (resulting from a C-to-T polymorphism) led to lower survival duration in patients with SQLC of the head and neck. The functional mechanism of DNMT3b in prognosis is still unclear. Unlike DNMT1, DNMT3b has been suggested to be site-selective in the regulation of aberrant gene silencing. DNMT3b expression has also been found to be essential for cancer cell survival by inhibiting apoptosis of tumour cells but not normal cells (Beaulieu et al, 2002). The results of in vitro studies by Geiman et al (2004) further indicate that $D N M T 3 b$ contributes to gene silencing by recruiting chromatin remodeling histone deacetylase.

In this study, we also found that the high level of $M B D 2$ expression was associated with a significant protective effect in male patients (HR, 0.40;95\% CI, 0.17-0.94) and SQLC patients (HR, 0.35; 95\% CI, $0.12-1.03$ ). MBD2 has been found to catalyse demethylation by directly removing methyl groups from 5methylcytosine residues in DNA (Bhattacharya et al, 1999). The results of our previous study (Zhu et al, 2004) suggest that MBD2 expression prevents age-related, sex-related, and smoking-induced hypermethylation. Several studies have demonstrated altered $M B D 2$ mRNA expression in various tumour tissue specimens, but the findings have been inconsistent. A significant reduction in the level of MBD2 mRNA expression was found in human colorectal and gastric tissues compared with nonmalignant tissues (Kanai et al, 1999), whereas elevated expression was reported in breast cancer (Billard et al, 2002). These differences may be explained by MBD2's dual functions (transcription repression and demethylation). To date, the specific role of MBD2 in NSCLC remains to be determined. In the present study, NSCLC tumour tissues had significantly lower levels of $M B D 2$ expression than did adjacent normal tissues (data not shown), suggesting that the effect of $M B D 2$ expression on clinical outcome may be related to demethylation. However, more conclusive evidence is needed.

Methylation varies as a function of age, sex, and smoking status (Ahuja and Issa, 2000; Toyooka et al, 2003). Therefore, it is plausible for our study to indicate that age, gender and smoking 
Table 3 Combined effect of DNMTI and DNMT3b, DNMTI and MBD2 or DNMT3b and MBD2 on survival stratified by age, sex, and histology

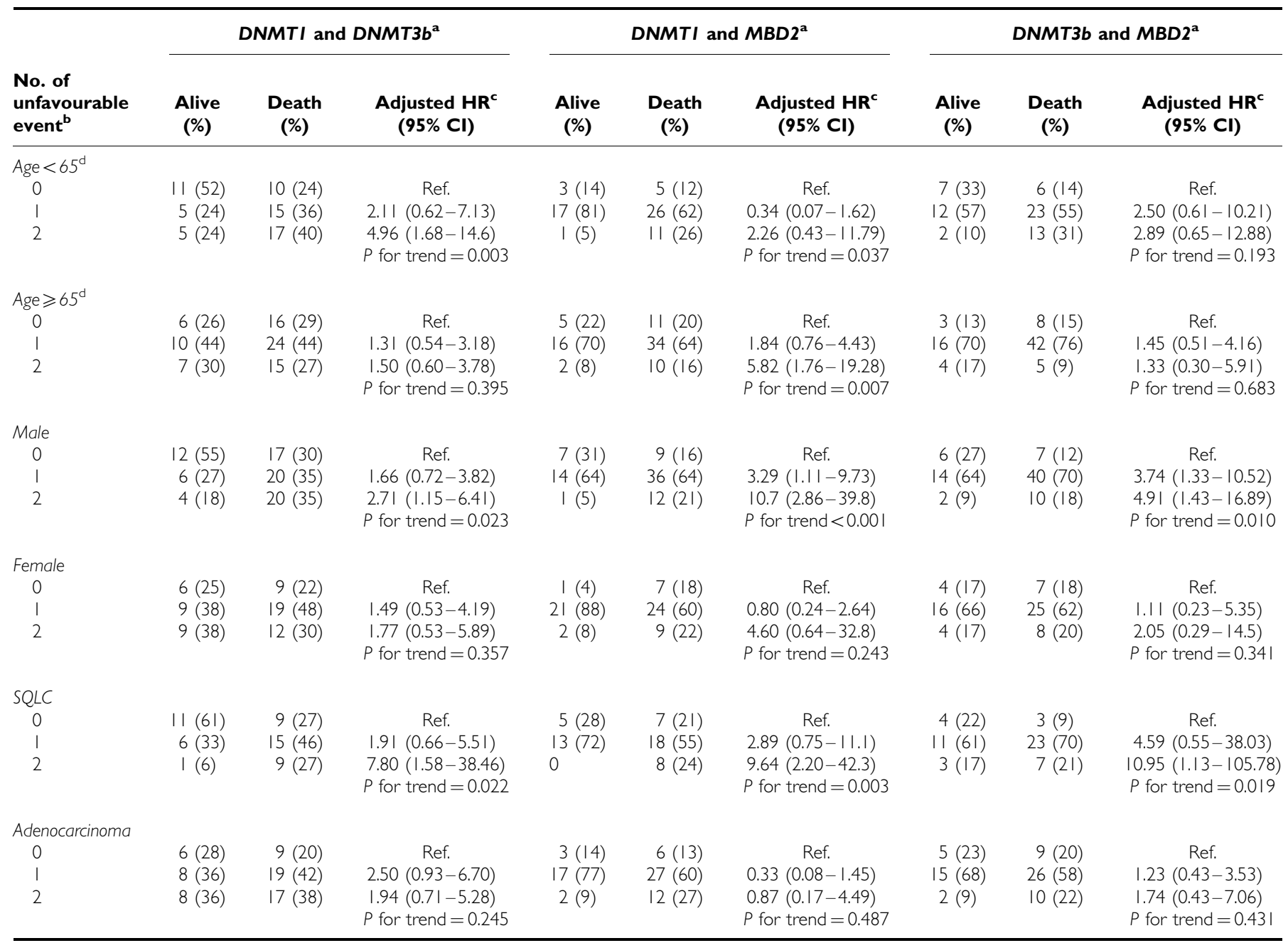

${ }^{a}$ Number of patients may not add up to the total number due to assay failure. ${ }^{b}$ Unfavourable factors were defined as high mRNA expression for DNMTI and DNMT3b or low mRNA expression for MBD2. ${ }^{C}$ Adjusted for age, sex, ethnicity, smoking status, tumour grade and clinical disease stage when appropriate. ${ }^{\mathrm{d} D i c h o t o m i s e d ~ u s i n g ~ t h e ~ m e d i a n ~ v a l u e ~}$ in all patients.

may modify the prognostic value of methylation-related genes in NSCLC patients. In addition, histologic tumour type-related differences in methylation have been documented in many genes, particularly tumour suppressor genes (Kim et al, 2004). Accordingly, our data revealed a histology-related prognostic significance of DNMT1 and MBD2 expression in NSCLC patients. To comprehensively evaluate the prognostic value of DNMT1, $D N M T 3 b$, and $M B D 2$, we performed a combined analysis. Our results suggest that these genes may be useful for determining prognosis in NSCLC patients, particularly in male patients and SQLC patients. Nevertheless, the results of our stratified and combined analyses should be interpreted cautiously because of the small sample size in each stratum and empirical cutoff point chooses. A larger study is needed to verify these findings.

To conclude, our findings further clarify the inconsistent findings of previous reports. We found that elevated DNMT1

\section{REFERENCES}

Ahuja N, Issa JP (2000) Aging, methylation and cancer. Histol Histopathol 15: $835-842$

Barrett JC, Oshimura M, Koi M (1986) Role of oncogenes and tumor suppressor genes in a multistep model of carcinogenesis. Symp Fundam Cancer Res 39: 45-56
mRNA expression was significantly associated with poor prognosis in NSCLC patients. DNMT3b and MBD2 had potential age-, sex-, and histology-related prognostic value in NSCLC patients, respectively. In addition, there is a significant combined effect of these three genes on overall survival. Therefore, these three genes could be useful in predicting clinical outcome in NSCLC patients and thus identifying patients who would benefit from aggressive therapy.

\section{ACKNOWLEDGEMENTS}

This work was supported by Grants CA 70907, CA 55769, and CA 111646 from the National Cancer Institute and DAMD 17-02-10706 from the US Department of Defense. 
Bhattacharya SK, Ramchandani S, Cervoni N, Szyf M (1999) A mammalian protein with specific demethylase activity for mCpG DNA. Nature 397: $579-583$

Billard LM, Magdinier F, Lenoir GM, Frappart L, Dante R (2002) MeCP2 and MBD2 expression during normal and pathological growth of the human mammary gland. Oncogene 21: 2704-2712

Biniszkiewicz D, Gribnau J, Ramsahoye B, Gaudet F, Eggan K, Humpherys D, Mastrangelo MA, Jun Z, Walter J, Jaenisch R (2002) Dnmt1 overexpression causes genomic hypermethylation, loss of imprinting, and embryonic lethality. Mol Cell Biol 22: 2124-2135

Chuang LS, Ian HI, Koh TW, Ng HH, Xu G, Li BF (1997) Human DNA(cytosine-5) methyltransferase-PCNA complex as a target for p21WAF1. Science 277: 1996-2000

Egger G, Jeong S, Escobar SG, Cortez CC, Li TW, Saito Y, Yoo CB, Jones PA, Liang G (2006) Identification of DNMT1 (DNA methyltransferase 1) hypomorphs in somatic knockouts suggests an essential role for DNMT1 in cell survival. Proc Natl Acad Sci USA 103: 14080-14085

Esteller M (2005) Aberrant DNA methylation as a cancer-inducing mechanism. Annu Rev Pharmacol Toxicol 45: 629-656

Geiman TM, Sankpal UT, Robertson AK, Zhao Y, Zhao Y, Robertson KD (2004) DNMT3B interacts with hSNF2H chromatin remodeling enzyme, HDACs 1 and 2, and components of the histone methylation system. Biochem Biophys Res Commun 318: 544-555

Girault I, Tozlu S, Lidereau R, Bieche I (2003) Expression analysis of DNA methyltransferases $1,3 \mathrm{~A}$, and $3 \mathrm{~B}$ in sporadic breast carcinomas. Clin Cancer Res 9: 4415-4422

Hoffmann MJ, Schulz WA (2005) Causes and consequences of DNA hypomethylation in human cancer. Biochem Cell Biol 83: 296-321

Issa JP, Vertino PM, Wu J, Sazawal S, Celano P, Nelkin BD, Hamilton SR, Baylin SB (1993) Increased cytosine DNA-methyltransferase activity during colon cancer progression. J Natl Cancer Inst 85: 1235-1240

Jemal A, Siegel R, Ward E, Murray T, Xu J, Thun MJ (2007) Cancer statistics, 2007. CA Cancer J Clin 57: 43-66

Jones PA, Laird PW (1999) Cancer epigenetics comes of age. Nat Genet 21: $163-167$

Kanai Y, Ushijima S, Nakanishi Y, Hirohashi S (1999) Reduced mRNA expression of the DNA demethylase, MBD2, in human colorectal and stomach cancers. Biochem Biophys Res Commun 264: $962-966$

Kim H, Kwon YM, Kim JS, Han J, Shim YM, Park J, Kim DH (2006) Elevated mRNA levels of DNA methyltransferase-1 as an independent prognostic factor in primary nonsmall cell lung cancer. Cancer 107: $1042-1049$

Kim JS, Kim H, Shim YM, Han J, Park J, Kim DH (2004) Aberrant methylation of the FHIT gene in chronic smokers with early stage squamous cell carcinoma of the lung. Carcinogenesis 25: $2165-2171$

Leonhardt H, Bestor TH (1993) Structure, function and regulation of mammalian DNA methyltransferase. Exs 64: 109-119

Lin RK, Hsu HS, Chang JW, Chen CY, Chen JT, Wang YC (2007) Alteration of DNA methyltransferases contributes to $5^{\prime} \mathrm{CpG}$ methylation and poor prognosis in lung cancer. Lung Cancer 55: 205-213

Lin X, Gu J, Lu C, Spitz MR, Wu X (2006) Expression of telomere-associated genes as prognostic markers for overall survival in patients with nonsmall cell lung cancer. Clin Cancer Res 12: 5720-5725

Luczak MW, Jagodzinski PP (2006) The role of DNA methylation in cancer development. Folia Histochem Cytobiol 44: 143-154
McCabe DC, Caudill MA (2005) DNA methylation, genomic silencing, and links to nutrition and cancer. Nutr Rev 63: 183-195

McCabe MT, Low JA, Daignault S, Imperiale MJ, Wojno KJ, Day ML (2006) Inhibition of DNA methyltransferase activity prevents tumorigenesis in a mouse model of prostate cancer. Cancer Res 66: 385-392

Newell-Price J, Clark AJ, King P (2000) DNA methylation and silencing of gene expression. Trends Endocrinol Metab 11: 142-148

Patra SK, Patra A, Zhao H, Dahiya R (2002) DNA methyltransferase and demethylase in human prostate cancer. Mol Carcinog 33: 163-171

Paz MF, Wei S, Cigudosa JC, Rodriguez-Perales S, Peinado MA, Huang TH, Esteller M (2003) Genetic unmasking of epigenetically silenced tumor suppressor genes in colon cancer cells deficient in DNA methyltransferases. Hum Mol Genet 12: 2209-2219

Rhee I, Bachman KE, Park BH, Jair KW, Yen RW, Schuebel KE, Cui H, Feinberg AP, Lengauer C, Kinzler KW, Baylin SB, Vogelstein B (2002) DNMT1 and DNMT3b cooperate to silence genes in human cancer cells. Nature 416: $552-556$

Robert MF, Morin S, Beaulieu N, Gauthier F, Chute IC, Barsalou A, MacLeod AR (2003) DNMT1 is required to maintain CpG methylation and aberrant gene silencing in human cancer cells. Nat Genet 33: 61-65

Robertson KD, Uzvolgyi E, Liang G, Talmadge C, Sumegi J, Gonzales FA, Jones PA (1999) The human DNA methyltransferases (DNMTs) 1, 3a and $3 \mathrm{~b}$ : coordinate mRNA expression in normal tissues and overexpression in tumors. Nucleic Acids Res 27: 2291 - 2298

Sato M, Horio Y, Sekido Y, Minna JD, Shimokata K, Hasegawa Y (2002) The expression of DNA methyltransferases and methyl-CpG-binding proteins is not associated with the methylation status of p14(ARF), p16(INK4a) and RASSF1A in human lung cancer cell lines. Oncogene 21: 4822-4829

Shames DS, Minna JD, Gazdar AF (2007) DNA methylation in health, disease, and cancer. Curr Mol Med 7: 85-102

Simons JW (1995) Genetic, epigenetic, dysgenetic, and non-genetic mechanisms in tumorigenesis. Crit Rev Oncog 6: $261-273$

Teodoridis JM, Strathdee G, Brown R (2004) Epigenetic silencing mediated by $\mathrm{CpG}$ island methylation: potential as a therapeutic target and as a biomarker. Drug Resist Updat 7: 267-278

Toyooka S, Maruyama R, Toyooka KO, McLerran D, Feng Z, Fukuyama Y, Virmani AK, Zochbauer-Muller S, Tsukuda K, Sugio K, Shimizu N, Shimizu K, Lee H, Chen CY, Fong KM, Gilcrease M, Roth JA, Minna JD, Gazdar AF (2003) Smoke exposure, histologic type and geographyrelated differences in the methylation profiles of non-small cell lung cancer. Int J Cancer 103: $153-160$

Vallbohmer D, Brabender J, Yang D, Schneider PM, Metzger R, Danenberg KD, Holscher AH, Danenberg PV (2006) DNA methyltransferases messenger RNA expression and aberrant methylation of $\mathrm{CpG}$ islands in nonsmall cell lung cancer: association and prognostic value. Clin Lung Cancer 8: 39-44

Wang L, Rodriguez M, Kim ES, Xu Y, Bekele N, El-Naggar AK, Hong WK, Mao L, Oh YW (2004) A novel C/T polymorphism in the core promoter of human de novo cytosine DNA methyltransferase 3B6 is associated with prognosis in head and neck cancer. Int J Oncol 25: $993-999$

Wu X, Amos CI, Zhu Y, Zhao H, Grossman BH, Shay JW, Luo S, Hong WK, Spitz MR (2003) Telomere dysfunction: a potential cancer predisposition factor. J Natl Cancer Inst 95: 1211-1218

Zhu Y, Spitz MR, Zhang H, Grossman HB, Frazier ML, Wu X (2004) Methyl-CpG-binding domain 2: a protective role in bladder carcinoma. Cancer 100: 1853-1858 